\title{
Adherencia al tratamiento en adolescentes diabéticos tipo 1 chilenos: una aproximación psicológica
}

\author{
Manuel Ortiz $\mathbf{P}^{1} \mathbf{a}$, Eugenia Ortiz $\mathbf{P}^{2}$. \\ Psychological factors associated to \\ patient's treatment compliance in \\ Chilean diabetic teenagers
}

Background: Treatment compliance among patients with type 1 diabetes mellitus, is low in $50 \%$ of diabetic teenagers, becoming a social and medical problem. Aim: To determine psycho-social factors associated to treatment compliance among Chilean diabetic type 1 teenagers. Patients and methods: A non experimental study of 61 diabetic teenagers (age 14.9 \pm 1.9 years, 37 male). The number of blood glucose determinations, socioeconomic level and practice of sports was measured. Psychological tests were applied to analyze self-efficiency, motivation of achievement, self-esteem and knowledge of the illness and its treatment. As a measure of patient compliance, glycosilated hemoglobin (HB1Ac) was measured. Results: Six patients had a good control of diabetes (HB1Ac <7\%), 24 had HB1Ac values between 7 and 8.9, and $31(51 \%)$ had values of $9 \%$ or more, considered as a poor diabetes control. The intensified insulin treatment scheme, the knowledge of the illness and its treatment and the sense of self-efficiency, were the factors associated with a better compliance with treatment. Teenagers of higher socio-economical levels had a better compliance with treatment. Conclusions: Fifty percent of Chilean diabetic teenagers in this sample had a poor control of the disease and the variable knowledge about the disease is the better predictor of patient compliance (Rev Méd Chile 2005; 133: 307-13).

(Key Words: Adolescent psychology; Diabetes mellitus, type 1; Patient compliance)

Recibido el 26 de mayo, 2004. Aceptado en versión corregida el 6 de enero, 2005.

Este estudio contó con el apoyo de los laboratorios Bayer y Roche, quienes suministraron los insumos para realizar el estudio.

${ }^{1}$ Facultad de Psicología, Universidad Mayor, Temuco.

${ }^{2}$ Programa de Formación de Endocrinología, Facultad de Medicina. Pontificia Universidad Católica de Chile.

apsicólogo, Magíster en Psicología.

Correspondencia a: Manuel Ortiz P. Fono: (45) 206336.

Fax: (45) 206 313. E-mail: msortiz@umayor.cl 
$\mathrm{L}_{\mathrm{a}}$ a falta de adherencia a los tratamientos es un problema altamente relevante en enfermedades crónicas como la diabetes, pues conlleva altos costos para el paciente y la sociedad, ya que a las secuelas físicas (enfermedad micro y macrovascular), se deben sumar costos por rehabilitación, pensiones de invalidez y pérdida de productividad ${ }^{1}$. Además, la adhesión al tratamiento determina su eficiencia y mejora la calidad de vida de las personas ${ }^{2}$.

La complejidad y cronicidad del tratamiento, sumado a los cambios biopsicosociales que ocurren durante la adolescencia, probablemente sobrepasen las competencias del adolescente para responder adecuadamente al tratamiento ${ }^{3}$. Consistente con esto, diversos estudios internacionales reportan que $50 \%$ de los adolescentes, con una enfermedad crónica, no adhieren completamente a su tratamiento ${ }^{4-8}$.

Diversos factores psicológicos, demográficos y de comportamiento han sido vinculados con la adherencia al tratamiento en adolescentes diabéticos. De las variables psicológicas, la autoeficacia percibida (creencia en las propias capacidades para organizar y ejecutar los cursos de acción requeridos para manejar situaciones futuras ${ }^{9}$ ), parecería mediar la relación entre responsabilidad y adherencia al tratamiento de adolescentes diabéticos ${ }^{10,11}$. Asimismo, se señala que la autoeficacia puede conducir a mejorar el control de las glicemias de los pacientes, debido a que correlaciona positivamente con las conductas de salud deseadas en el tratamiento de la diabetes. Además, incrementaría la habilidad del paciente para guiar su tratamiento, de modo independiente al equipo médico, lo que a su vez podría conllevar menos visitas a los centros de salud ${ }^{12}$.

Otros autores ${ }^{13}$, mencionan que una baja autoestima y por ende, mayor dependencia social, son factores más comunes entre adolescentes diabéticos que entre niños y adolescentes sin la enfermedad y de la misma edad, lo que de alguna forma podría sugerir como consecuencia de la enfermedad un descenso en la autoestima de quien la padece. Se puede esperar que aquellos adolescentes diabéticos con una autoestima más elevada, presenten mayor adherencia al régimen de salud que aquellos adolescentes con una baja autoestima $^{14}$.
Se estima que la motivación de logro, tiene una influencia en la adherencia mayor que los factores demográficos y metabólicos ${ }^{15}$. Se sugiere que el conocimiento (información) resulta esencial para la adherencia del paciente, no obstante, la información por sí sola, parecenía no ser suficiente para promover los cambios conductuales requeridos como parte del manejo de la enfermedad ${ }^{16}$. A pesar de esto, se ha encontrado en adolescentes diabéticos que presentan un pobre control de su diabetes, una carencia de conocimientos sobre su enfermedad y su tratamiento ${ }^{17}$. Sin embargo, diabéticos con un adecuado grado de información sobre la enfermedad y su tratamiento, también pueden tener un deficiente control de su condición ${ }^{18}$.

De los factores demográficos, Johnson et $\mathrm{al}^{19}$, reportan que entre jóvenes diabéticos cuyas edades fluctúan entre los 6 y 19 años, la mejor adherencia se produce entre aquel grupo de menor edad. A su vez, Bond et $\mathrm{al}^{20}$, señalan que en el grupo de diabéticos cuyas edades oscilan entre los 10 y 19 años, la adhesión al tratamiento es más pobre entre los de mayor edad.

El nivel socioeconómico del paciente generalmente ha sido asociado con la adherencia al tratamiento, reportándose una correlación directa entre ambas variables ${ }^{5}$.

Variables de comportamiento como el tratamiento insulínico seguido por el paciente (convencional $\mathrm{v} / \mathrm{s}$ intensificado), la frecuencia de glicemias realizadas diariamente ${ }^{21}$ y la práctica de actividad física ${ }^{5}$, son variables que han sido vinculadas con la adherencia al tratamiento y el grado de control metabólico de los adolescentes diabéticos. Aquellos adolescentes que emplean el tratamiento de múltiples dosis, presentan una mayor adherencia al tratamiento y un mejor control metabólico que aquellos que siguen el esquema convencional ${ }^{21}$. Es posible creer que aquellos adolescentes que realizan mayor cantidad de controles diarios de glicemia, demuestren tener un mejor control de su diabetes, debido a que disponer de mayor información sobre su grado de ajuste metabólico, permitiría tomar mejores decisiones respecto a, por ejemplo, qué dosis de insulina inyectar, o qué tipo de alimento ingerir. Asimismo, se espera que los adolescentes que realizan actividad física, logren tener un mejor control metabólico ${ }^{22}$. 
El objetivo general de este estudio, fue determinar si los factores psicológicos, demográficos y de comportamiento se asocian a la adherencia al tratamiento, en una muestra de adolescentes diabéticos tipo 1 chilenos.

\section{MATERIAL Y MÉTODO}

a) Diseño: El diseño de este estudio es no experimental, correlacional, transversal, pues no se manipulan variables independientes y se pretende evaluar la asociación de éstas en un momento dado.

b) Participantes. Se seleccionó aleatoriamente una muestra de sujetos de los registros de la Fundación de Diabetes Juvenil de Chile (FDJ), los cuales fueron invitados a participar como voluntarios por medio del envío de una carta de consentimiento informado, tanto para los adolescentes como para sus padres. Se seleccionó en base a los siguientes criterios: a) consentimiento informado para participar en el estudio; b) los sujetos han sido diagnosticados con diabetes mellitus tipo 1; c) diagnóstico realizado con una anterioridad igual o superior a un año; d) edad entre 12 y 18 años. Para resguardar y cautelar el bienestar de los participantes, se cumplieron los principios éticos que dicta la American Psychological Association ${ }^{23}$ respecto al trabajo con humanos. La recolección de datos se efectuó en la sede de la FDJ en Santiago. Se administraron test y cuestionarios psicológicos ${ }^{24-26}$ y se efectuó un examen de hemoglobina glicosilada (Hb1Ac), examen realizado por una enfermera universitaria. Se tuvo al alcance productos para el auxilio de episodios leves y moderados de hipoglicemia.

c) Estadística. Frecuencias y datos descriptivos fueron computados usando el software SPSS. Se efectuaron análisis de correlación y se ejecutó un análisis de regresión múltiple, por medio del método de pasos sucesivos. Para las variables nivel socioeconómico de los participantes y su grado de conocimiento (información), se ejecutó un análisis de varianza de una vía (ANOVA) y comparaciones analíticas planificadas.

En este estudio, los niveles de HB1Ac fueron utilizados como medida de la adherencia al tratamiento de los adolescentes diabéticos, tal como ha sido realizado en estudios previos ${ }^{27}$. Por tanto, aquellos adolescentes que obtuvieron menores porcentajes de $\mathrm{Hb} 1 \mathrm{Ac}$, fueron categorizados como sujetos más adherentes, siguiendo la clasificación establecida por el Diabetes Control and Complications Trial Research Group (DCCT) ${ }^{21}$.

Los datos fueron analizados con un nivel de significación de $5 \%$ y $1 \%$, respectivamente.

\section{RESULTADOS}

La Tabla 1, muestra que 60,7\% de los participantes son mujeres y $39,3 \%$ hombres, el promedio de edad fue 14,9 años $(\mathrm{SD}=1,88)$. El $82 \%$ de los participantes tiene dos años o más con la enfermedad. El nivel socioeconómico tendió a ser heterogéneo.

\section{Tabla 1. Información demográfica y de comportamiento de los participantes}

\begin{tabular}{|lcc|}
\hline Características & $\mathrm{n}$ & $\%$ \\
de los participantes & & \\
\hline Sexo & & \\
$\quad$ Mujeres & 37 & 60,7 \\
Hombres & 24 & 39,3 \\
Años con diabetes & & \\
$\quad$ Menos de 2 años & 11 & 18 \\
$\quad$ Más de 2 años & 50 & 82 \\
Nivel socioeconómico & & \\
Bajo & 20 & 32,8 \\
$\quad$ Medio & 23 & 37,7 \\
Alto & 18 & 29,5 \\
Control de glicemia visual & & \\
$\quad$ Realiza & 22 & 36,1 \\
No realiza & 39 & 63,9 \\
Control de glicemia con máquina & \\
$\quad$ Realiza & 49 & 80,3 \\
No realiza & 12 & 19,7 \\
Controla glicemia con ambos métodos & \\
Sí & 10 & 16,4 \\
No & 51 & 83,6 \\
Tratamiento & & \\
$\quad$ Esquema intensificado & 32 & 52,5 \\
Esquema convencional & 29 & 47,5 \\
Deporte & & \\
Realiza & 43 & 70,5 \\
No realiza & 18 & 29,5 \\
\hline
\end{tabular}


Los participantes, reportaron haber realizado, en promedio, 3,3 controles de glicemia diaria. El 36,1\% había empleado métodos visuales mientras que $80,3 \%$ lo hacía con máquinas de glicemia, 16,4\% empleaba ambos procedimientos. El 52,5\% empleaba tratamiento insulínico intensificado y $70,5 \%$ reporta haber realizado actividad física en forma regular.

Respecto al grado de control metabólico, 6 jóvenes $(9,8 \%)$ presentaron un buen control metabólico con valores bajo 7\%, 24 adolescentes $(39,4 \%)$ obtuvieron índices satisfactorios (HB1Ac entre 7 y $8,9 \%)$ y 31 participantes (50,8\%) demostraron un pobre control de su diabetes (valores sobre 8,9\%) (según valores de referencia del DCCT) ${ }^{21}$.

Tal como se muestra en la Tabla 2, las variables psicológicas conocimiento sobre la diabetes y su tratamiento $(\mathrm{r}=-0,398, \mathrm{~N}=61, \mathrm{p}<0,001)$ y la percepción de autoeficacia, $(r=-0,282, N=61, p$ $<0,05)$, se asociaron de modo significativo y en la dirección esperada con la adherencia al tratamiento de los adolescentes diabéticos. Por tanto, era esperable que aquellos adolescentes que tenían mayor conocimiento sobre su enfermedad y mayor sentido de autoeficacia presentaran mayor adherencia al tratamiento. Las variables autoestima $(\mathrm{r}=-0,208, \mathrm{~N}=61, \mathrm{p}=0,108)$, y motivación de logro $(r=-0,239, N=61, p=0,064)$, se encontraron en la dirección esperada, no obstante, su asociación resultó ser estadísticamente no significativa.

En la Tabla 3, se observa que el esquema de tratamiento intensificado se asoció de modo inverso con la adherencia al tratamiento $(r=-0,378, N=$ $61, \mathrm{p}<0,05$ ). La variable nivel socioeconómico de los adolescentes diabéticos, se asoció con la adherencia en la misma dirección $(r=-0,363, N=$ $61, \mathrm{p}<0,001)$. Al comparar los niveles socioeconómicos (alto, medio y bajo) de los participantes, se apreciaron diferencias en los respectivos niveles de adherencia al tratamiento $(\mathrm{F}=5,421, \mathrm{p}<0,001)$.

Tabla 2. Factores psicológicos asociados a la adherencia al tratamiento: Análisis de correlación

\begin{tabular}{|lccccc|}
\hline & Hb1Ac & $\begin{array}{c}\text { Conocimiento } \\
\text { de diabetes }\end{array}$ & Autoestima & $\begin{array}{c}\text { Motivación } \\
\text { de logro }\end{array}$ & Autoeficacia \\
\hline Hb1Ac & 1,000 & $-0,398^{* *}$ & $-0,208$ & $-0,239$ & $-0,282^{*}$ \\
Conocimiento de diabetes & & 1,000 & 0,158 & $0,332 *$ & 0,115 \\
Autoestima & & 1,000 & 0,099 & $0,293^{*}$ \\
Motivación de logro & & & & 1,000 & 0,055 \\
Autoeficacia & & & & 1,000 \\
\hline
\end{tabular}

${ }^{*}$ Correlación es significativa al 0,01. *Correlación es significativa al 0,05.

Tabla 3. Asociación entre la adherencia al tratamiento y las variables demográficas y de comportamiento

\begin{tabular}{|lccccccc|}
\hline & Hb1Ac & $\begin{array}{c}\text { Esquema } \\
\text { intensificado }\end{array}$ & $\begin{array}{c}\text { Controles } \\
\text { glicemia }\end{array}$ & Edad & $\begin{array}{c}\text { Años } \\
\text { diabetes }\end{array}$ & Deporte & $\begin{array}{c}\text { Nivel } \\
\text { socio } \\
\text { económico }\end{array}$ \\
\hline Hb1Ac & 1,000 & $-0,378^{* *}$ & $-0,239$ & 0,015 & 0,117 & $-0,122$ & $-0,363^{* *}$ \\
Esquema intensificado & & 1,000 & $0,442^{* *}$ & 0,079 & $-0,023$ & 0,040 & 0,164 \\
Controles glicemia & & & 1,000 & $-0,286^{*}$ & $-0,164$ & $0,270^{*}$ & 0,130 \\
Edad & & & 1,000 & $0,475^{* *}$ & $-0,256^{*}$ & 0,078 \\
Años diabetes & & & & 1,000 & $-0,102$ & $-0,104$ \\
Deporte & & & & & 1,000 & 0,064 \\
Nivel socio económico & & & & & & & 1,000 \\
\hline
\end{tabular}

${ }^{* *}$ Correlación es significativa al 0,01. ${ }^{*}$ Correlación es significativa al 0,05. 
Los contrastes ortogonales realizados aportan evidencia de diferencias estadísticamente significativas en el nivel de adherencia al tratamiento, entre los participantes provenientes del estrato socioeconómico más bajo y los de los niveles medio $\mathrm{y}$ alto $(\mathrm{p}<0,05)$. Además, fue posible determinar que los participantes pertenecientes al estrato socioeconómico medio y alto, también presentaron diferencias estadísticamente significativas en su adherencia al tratamiento ( $\mathrm{p}<0,001)$. Por tanto, aquellos adolescentes de mayor nivel socioeconómico podrían presentar mayor adherencia al tratamiento que los del estrato medio y éstos, a su vez, mayor que los de bajo nivel socioeconómico.

Aquellos participantes con bajo, medio y alto grado de conocimiento demostraron diferencias en sus niveles de adherencia $(F=6,580, p<0,001)$. Estas diferencias se dieron entre los participantes de bajo y mediano conocimiento con aquellos de alto conocimiento $(p<0,001)$. No se encontraron diferencias entre los adolescentes con bajo y medio grado de conocimiento $(p=0,315)$. Este resultado sugiere que para adherir bien al tratamiento sería necesario contar con alto grado de conocimiento sobre el tratamiento.

En la Tabla 4, se muestra el resultado del análisis de regresión lineal. De los predictores ingresados al modelo, el nivel de conocimiento que tienen los adolescentes sobre la diabetes y su tratamiento explicó $16,8 \%$ de la varianza $\left(\mathrm{R}^{2}=\right.$ $0,168, p<0,01$ ), el esquema de tratamiento intensificado dio cuenta del 9,9\% de la varianza $\left(\mathrm{R}^{2}=\right.$ $0,267, \mathrm{p}<0,01)$ y el grado de autoeficacia percibida $7,4 \%$ de la varianza $\left(\mathrm{R}^{2}=0,341, \mathrm{p}<0,014\right)$. Este resultado podría aportar en la predicción de la adherencia al tratamiento, sugiriendo que el factor que mejor predice el comportamiento adherente en este estudio fue el conocimiento que tenían los adolescentes sobre su enfermedad, seguido por el uso del esquema intensificado y el grado de autoeficacia percibido.

\section{Discusión}

Los hallazgos de esta investigación demuestran que $50 \%$ de adolescentes diabéticos, al momento de ser estudiados, adhieren pobremente a su tratamiento. Tales cifras resultan alarmantes, si se considera que estos adolescentes, en el mediano y largo plazo, se encaminan a sufrir las consecuencias asociadas a un deficiente control, con la consecuente pérdida de fuerza laboral para el país y carga para el Estado en materia sanitaria.

De los factores psicológicos, la variable conocimiento es la que permite predecir mejor la adherencia al tratamiento. De los participantes del estudio, aquellos con mayor conocimiento presentaron mejor adherencia al tratamiento que aquellos con un conocimiento medio y bajo. No se encontraron diferencias entre sujetos de bajo y medio conocimiento, lo que podría sugerir que para adherir adecuadamente al tratamiento, sería necesario un nivel de conocimiento elevado. Un alto grado de conocimiento podría favorecer un adecuado cumplimiento del tratamiento, por ejemplo, se podría decidir acertadamente cómo modificar dosis de insulina, reconocer síntomas de hipo e hiperglicemia y saber cómo manejarlos, entre muchas conductas esperables. Este resultado refuerza la tesis respecto a la importancia de la educación al paciente diabético, ya que como se ha mencionado, la educación puede reducir en $80 \%$ las complicaciones asociadas a un pobre control de la diabetes ${ }^{28}$.

La variable autoeficacia resultó ser predictora de la adherencia al tratamiento. Es posible pensar que un adolescente diabético cuyo sentido de autoeficacia sea elevado, podría ser capaz de motivarse y emprender conductas saludables para mantener su estado de bienestar. Por otra parte, un adolescente que ha sido exitoso en su control de la diabetes, podría experimentar una fuerte sensación de dominio sobre su salud y su autocui-

Tabla 4. Predictores de adherencia

\begin{tabular}{|lccccc|}
\hline Variable & $\mathrm{R}^{2}$ & $\mathrm{~B}$ & $\mathrm{~S}$ & $\mathrm{t}$ & $\mathrm{P}$ \\
\hline Nivel de conocimiento & 0,168 & $-0,114$ & $-0,328$ & $-3,452$ & 0,01 \\
Esquema intensificado & 0,267 & $-0,127$ & $-0,342$ & $-2,800$ & 0,01 \\
Autoeficacia percibida & 0,341 & $-0,018$ & $-0,274$ & $-2,533$ & 0,01 \\
\hline
\end{tabular}


dado. Se ha informado que el sentir dominio sobre un área específica, constituye una de las principales fuentes de la autoeficacia ${ }^{29}$. El nivel socioeconómico de los participantes, resultó ser una variable que marca diferencias en el grado de control metabólico. De este modo, aquellos adolescentes provenientes de estratos socioeconómicos más altos, tuvieron mejor adherencia que los adolescentes de estrato medio, los que a su vez, también presentaron mejor control que los participantes del estrato más bajo. El pertenecer a un alto nivel socioeconómico facilitaría el acceso a mejores prestaciones de salud, disponer de mejores métodos de control metabólico, realizar mayor cantidad de controles de glicemia al día, costear insulinas que favorecen un mejor control metabólico (insulinas rápidas y ultra rápidas versus insulinas lentas) y tener una dieta acorde a las demandas del régimen, además de tener mayor facilidad para acceder a diferentes medios de información (internet, libros, revistas) respecto a la enfermedad y su tratamiento.

Aquellos adolescentes diabéticos chilenos que tienen acceso al tratamiento insulínico intensificado, presentaron mejor adherencia al tratamiento. Este resultado es una evidencia más respecto de la mayor eficacia de esta modalidad de tratamiento en la adherencia y el control metabólico de la diabetes, tal como es señalado por el DCCT ${ }^{21}$. Tomando en referencia este resultado, parecería deseable, por una parte, estimular la prescripción de este tratamiento y por otra, garantizar el abastecimiento del arsenal farmacológico con insulinas e insumos que permitan la prescripción del tratamiento insulínico intensificado. No obstante este hallazgo, se debe ser cauteloso en su

\section{REFERENCIAS}

1. Maldonato A, Bloise D, Ceci M, Fraticelu E, Falucca F. Diabetes Mellitus: Lessons from patient education. Patient Educ Couns 1995; 26: 55-66.

2. Hidalgo C, CARRasco E. Salud familiar: Un modelo de atención integral en la atención primaria. Santiago: Ediciones Universidad Católica de Chile. 1999.

3. Kyngäs H, KroL M, DuFfy T. Conceptual analyses of concept compliance. J Clin Nurs 2000; 9: 5-12. interpretación, y no rotular a dicho factor como la panacea para el tratamiento de la diabetes, ya que pese a que este esquema de tratamiento tiende a imitar la acción fisiológica del páncreas, si no es bien empleado, ya sea por desinformación o por negligencia del paciente, es probable que no conduzca a los resultados esperados.

Este estudio cuenta con limitaciones, entre las cuales se puede señalar el tamaño de la muestra $(n=61)$. Es necesario apuntar que los participantes fueron aquellos que accedieron a participar en el estudio, por tanto, este estudio sería representativo sólo de los adolescentes que cumplieron con los criterios de selección informados. Además se realizó el cálculo post-hoc de la potencia del diseño y de la magnitud del efecto, estimándose valores de 0,58 y 0,40 respectivamente, con un nivel de significación de 0,05. Estos valores son aceptables y consistentes con estudios previos.

De este estudio se desprenden futuras líneas de investigación. Considerando la complejidad y cronicidad del tratamiento, es interesante cuestionarse la estabilidad de la adherencia que pueden presentar los adolescentes diabéticos, pues, al parecer, la mayoría de las conductas de salud son inestables en el tiempo, tendiendo a variar continuamente ${ }^{30}$. En el caso de la diabetes en adolescentes, se han reportado resultados controversiales, dando cuenta algunos estudios de fluctuaciones drásticas en los niveles de hemoglobina, y en otros de desviaciones no significativas en términos de mantener un nivel aceptable de control metabólico ${ }^{31}$. Por tanto, parece interesante cuestionarse en tomo a la estabilidad de la adherencia de los adolescentes diabéticos chilenos, estudio que se está llevando a cabo en este momento por el autor principal del estudio.

4. Adams S, PiLl R, Jones A. Medication, chronic illness and identity: The perspective of people with asthma. Soc Sci Med 1997; 45: 189-201.

5. Chigier E. Compliance in adolescent with epilepsy or diabetes. J Adolesc Health 1992; 13: 3759.

6. Hentinen M, Kyngäs H. Compliance of young diabetic's with health regimens. J Adv Nurs 1992; 17: 530-6.

7. KYNGÄS H. Diabetes: An adolescent's perspective. J Adv Nurs 1995; 22: 941-7. 
8. LAGRECA AM. Issues in adherence with pediatrics regimens. J Pediatr Psychol 1990; 15: 423-36.

9. BANDURA A. Exercise of personal agency trough the self-efficacy mechanism. En: Schwarzer R, ed. Self-efficacy: Trough control of action. Washington, DC: Hemisphere 1992.

10. KNeCKT MC, Syrjala AM, LaukKanen P, KnuUtTila ML. Self-efficacy as a common variable in oral health behavior and diabetes adherence. Eur J Oral Sci 1999; 107: 89-96.

11. Ott J, Greening L, Palardy N, Holderby A, Debell W. Self-efficacy as a mediator variable for adolescents' adherence to treatment for insulin-dependent diabetes mellitus. Child Health Care 2000; 29: 47-63.

12. ANDERSON R. The diabetes empowerment scale: A measure of psychosocial self-efficacy. Diabetes Care 2000; 11: 519-26.

13. Grey M, Davidson M, Boland E, Tamborlane W. Clinical and psychosocial factors associated with achievement of treatment goals in adolescents with diabetes mellitus. J Adolesc Health 2001; 28: 377-85.

14. Lo R. Correlates of expected success at adherence to health regimen of people with IDDM. J Adv Nurs 1999; 30: 418-24.

15. Miler-Johnson S, Emery R, Marvin R, Ciarke W, LOVINGER R, MARTIN M. Parent-child relationships and the management of insulin-dependent diabetes mellitus. J Consult Clin Psychol 1994; 62: 603-10.

16. BleEsing-Moore J. Does asthma education change behavior? To know is not to do. Chest 1996; 109: 9-11.

17. Burroughs TE, Pontius SL, Santiago JV. The relationship among six psycho-social domains, age, health care adherence, and metabolic control in adolescents with IDDM. Diabetes Educ 1993; 19: 396-402.

18. BARRY K. Patient self-medication: An innovative approach to medication teaching. J Nurs Care Qual 1993; 8: 75-82.
19. Johnson S, Freund A, Silverstein J, Hansen C, MALONE J. Adherence-health status relationship in childhood diabetes. Health Psychol 1990; 9: 60631.

20. Bond G, Aiken L, SomersviLe S. The health belief model and adolescents with insulin-dependent diabetes mellitus. Health Psychol 1992; 11: 190-8.

21. The Diabetes Control and Compucations Trial ReSEARCH GROUP. The effect of intensive treatment of diabetes on the development and progression of long-term complications in insulin-dependent diabetes mellitus. N Engl J Med 1993; 329: 977-86.

22. Fundación de Diabetes Juvenil de Chile. Vivir mejor. Manual ilustrado para el control de la diabetes con uso de insulina. Santiago: Antártica Quebecor S.A. 2000.

23. American Psychological Association. Ethical principles of psychologists and code of conduct. Am Psychol 1992; 47: 1597-611.

24. COOPERSMTTH S. A method for determining types of self-esteem. J Abnorm Soc Psychol 1959; 59: 8794.

25. Sherer M, Maddux J, Mercandante B, Prentice-Dunn S, Jacobs B, Rogers R. The self efficacy scale: Construction and validation. Psychol Rep 1982; 51: 663-71.

26. EDwards AL Personal Preference Schedule. Manual. New York N.Y: Psychological Corporation. 1959.

27. Kyngäs H. Compliance of adolescents with diabetes. Int Pediatr Nurs 2000; 15: 260-7.

28. Chantelau E, Schiffers T, Schütze J, Hansen B. Effect of patient-selected intensive insulin therapy on quality of life. Patient Educ Couns 1997; 30: 167-73.

29. Feuerstein M, Labbè E, Kuezmierczyk A. Health psychologic: A psychobiological perspective. New York: Plenum Press. 1986.

30. TAYLOR S. Health psychology. New York: Random House. 1986. 